\title{
Toward a comprehensive OSCM research methodology: The role of design science
}

\begin{abstract}
Research in Operations and Supply Chain Management (OSCM) field is widespread and applied to various sectors of industry and services. Despite its benefits, the diversity of the worldviews of OSCM research applications and the preferences regarding the choice of methodologies by the researchers sometimes cause unnecessary complexities in converging these efforts toward advancing theory and practice. It has been argued that the application of multi-methods could facilitate obtaining a common ground through which OSCM researchers could make more effective and robust contributions in binding theory and practice. There is, however, a lack of a unifying research methodology so that OSCM research could effectively link between theory and practice and move toward more coherence of research in the OSCM field. In this article, we introduce the application of design science research methodology and the use of multi-methods approach in this methodology to draw a road map for more coherent future research attempts in the domain of OSCM. As an example, the behavioral causes of the bullwhip effect are examined and illustration is made on how the dispersed but growing research efforts in this area could be aligned into drawing a behavioral theory of the ordering preferences of decision makers in supply chains and in organizations.
\end{abstract}

Keywords: Design science; multi-methods; operations and supply chain management 


\section{Toward a comprehensive OSCM research methodology: The role of design science}

Despite the increasing amount of research and publications by OSCM researchers in various fields of study from behavioral operations to service operations, there is a consensus among the OSCM community that the global OSCM research should address the "isolation in methodology" and "disconnection from practice" (Sodhi \& Tang, 2014). It has also been argued that OSCM research, at its current state, lacks a sufficient validity for managerial applications (Singhal \& Singhal, 2012a). Parallel to the quests in general management research (van Aken, 2004, 2005), OSCM researchers are encouraged to take a leap beyond the premises of mere theory testing to actual theory building by problem solving (Holmström, Ketokivi, \& Hameri, 2009; Singhal, Sodhi, \& Tang, 2014).

The idea of successful scientists in OSCM research being good problem solvers, observers of real-world problems, and also professional theorists is widely acknowledged by the OSCM

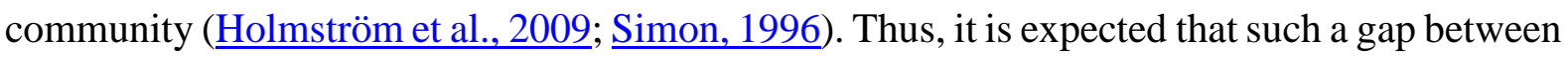
theory and practice in global OSCM research be filled by OSCM scholars through a proper research methodology. Such a methodology should reach beyond the geographical boundaries of research and be applied extensively in almost any context of the OSCM studies to produce and transfer knowledge that serves the purposes of both academia and practice.

In the current study we examine this problem through the prism of design science research methodology that aims at making connections between problem solving and theory-building efforts in research. We use the three cycles of relevance, rigor, and design to explain how new theories could emerge from real-world OSCM problems faced by managers and organizations. Specifically for the cycle of rigor, we investigate the possibility of using multi-methods to help reaching a desirable design solution and subsequently an applicable theory. We then apply the 
proposed framework to the behavioral operations context and provide guidelines into how the design science methodology could enhance the quality of research using multi-methods in this context.

\section{BACKGROUND}

\section{Design science}

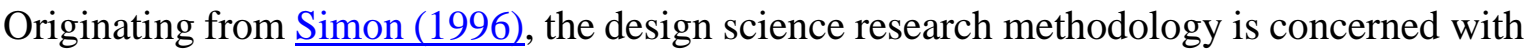
purposeful building and evaluation of artificial objects and phenomena. The latter are associated with acquiring new properties by a system to eliminate the existing effectiveness/efficiency issues within this system. Defining the mission of design science, $\underline{\text { van }}$ Aken (2004) stresses its focus on the development of scientific knowledge in order to support the design of new artifacts (e.g., theories, frameworks, models, methods) or the development of the existing artifacts by professionals. Thus, design science looks for developing an artifact to solve a problem in practice, otherwise it cannot be construed as a design science methodological approach. Design science has been widely adopted in the fields of Information Systems and Computer Science where it was reported to assist in understanding, explaining and frequently improving the behavior of existing systems by creating innovative and unique artifacts in a well-defined manner (Hevner, March, Park, \& Ram, 2004) or by analyzing the application of the proposed artifacts (March \& Smith, 1995). The design science research methodology has been also adopted in the domains of decision support systems (March \& $\underline{\text { Smith, 1995), information systems (Hevner et al., 2004), decision sciences (Holmström et al., }}$ 2009), and recently, in the domain of supply chain management, and specifically supply chain service triads ( Finne \& Holmström, 2013).

In the domain of social sciences, artifacts such as theories, frameworks, models, methods and instantiations are considered to be the major outputs of the design process (Gregor, 2006; 
Hevner et al., 2004; March \& Smith, 1995). In the context of OSCM research, contributing to theory or developing new theories is the desirable artifact, but this is considered to be an area in which OSCM research is falling behind considerably compared to other fields of management (Schroeder, 2008; Walker, Chicksand, Radnor, \& Watson, 2015). To guide theory development and its evaluation/justification, we adopt a three-phased methodology for conducting the design science research as suggested by Hevner et al. (2004) and Hevner (2007). Similar to the methodology proposed by Holmström and Romme (2012) in the context of Operations Management, it suggests a more detailed view on the artifact development and evaluation/justification process, representing it as three interrelated cycles of research activity: relevance cycle, rigor cycle and design cycle (Figure 1). The relevance cycle covers the tasks of aligning the requirements of contributing to an existing theory or developing a new theory according to the practical needs of people/organizations in a specific domain of study and/or in a specific industry/service sector. The design cycle assures the direct and feedback relationship between the theory development and the justification/evaluation steps. The rigor cycle provides the necessary methodological grounding for all the stages of the design process. Here we argue the use of multi-method approaches, which could provide a better means of achieving the theory development process for OSCM researchers. 


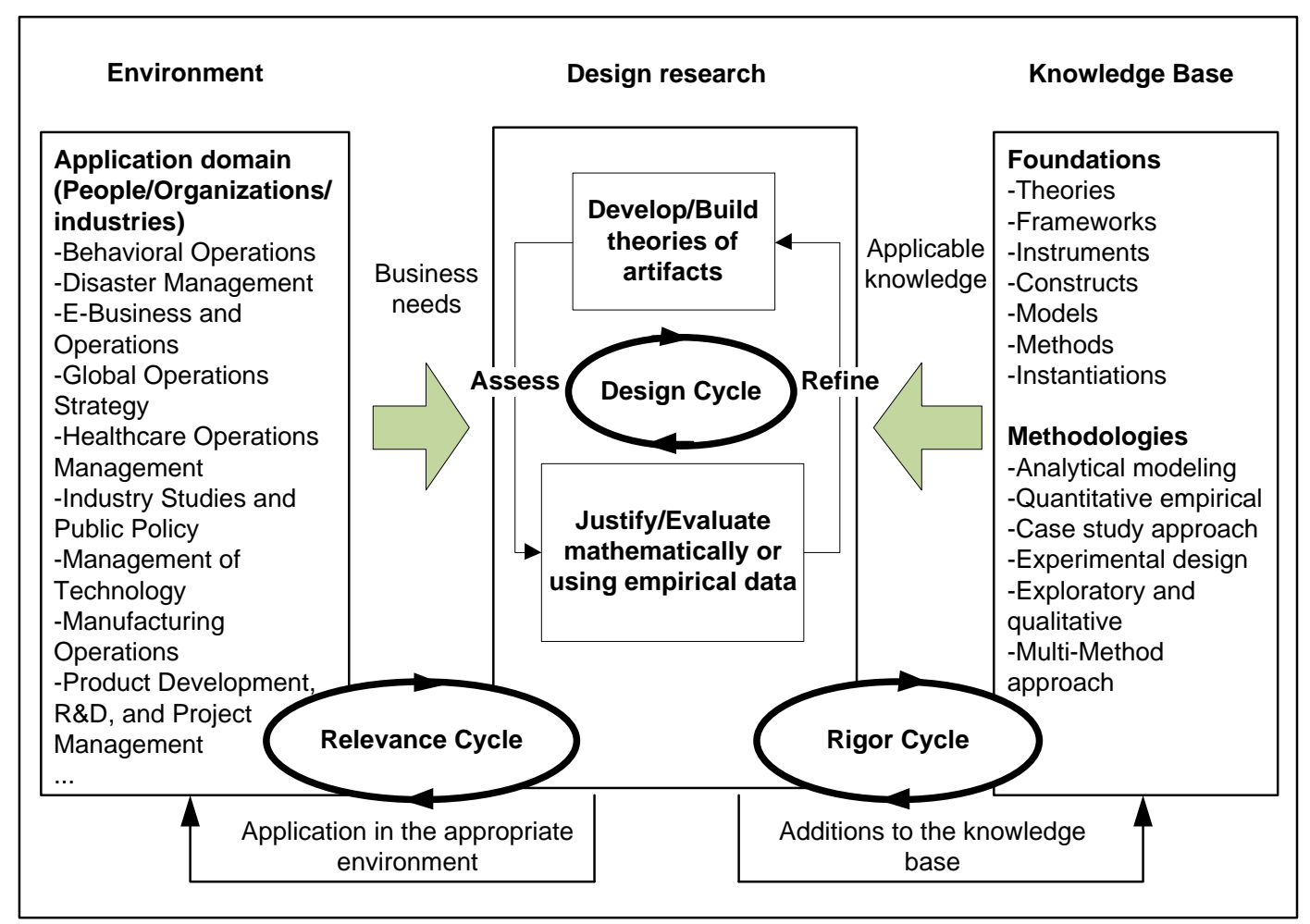

Figure 1 The proposed design science research methodology for global OSCM research

\section{Multi-methods}

Multi-methods apply multiple problem solving techniques from the same or different disciplines and are ideal for studying a phenomenon and understanding its complexities (Boyer \& Swink, 2008; Sanders \& Wagner, 2011). The increased rigor and reliability of adopting multi-methods results in "greater insights into research problems, reduction in the myopic, disciplined-based perspective, and greater potential for innovative SCM [Supply Chain Management] breakthroughs" (Sanders and Wagner (2011), p. 318). Choi, Cheng, and Zhao (2016) define multi-methods approach in OSCM studies as “... an approach for OM research in which at least two distinct $\mathrm{OM}$ research methods are employed nontrivially to meet the research goals". They subsequently elaborate on their discussions on multi-methods by identifying the two-by-two combinations of analytical modeling, quantitative empirical, and case study research methods (based on the classifications of Sodhi and Tang (2014)) and their application to OSCM studies. In addition to this classification, we will also include 
exploratory and qualitative multi-method as proposed by Singhal and Singhal (2012a) and $\underline{\text { Singhal and Singhal (2012b) }}$ to be one of the main players in our design science methodology for theory building purposes.

\section{PROPOSED RESEARCH METHODOLOGY}

\section{Relevance and design cycles}

Despite the critical role that developments in OSCM practices have played in managing organizations, "practice-driven academic research" has rarely initiated these advancements (Singhal \& Singhal, 2012a). In a similarly, most of the research conducted in OSCM's domain fail to include and address the practicality required by managers to apply the outcomes to their organizations (Singhal et al., 2014).

Perhaps, the main reason behind the lack of practicality of a research could be traced to the reluctance of researchers to make their insights presentable to real-word situations and industry decision makers, and this could be rooted in their original view of academic knowledge base and practice being two separate realms that cannot be articulated and converged toward the same end (Gulati, 2007). The current design science research methodology however justifies that the relevance cycle should be considered as a critical part of theory development and theory building in all areas of the OSCM research domain.

The relevance cycle consists of the first two phases of means-end analysis (Simon, 1996) in OSCM research proposed by Holmström et al. (2009); i.e., solution incubation and solution refinement. In brief, the solution incubation phase is concerned with defining a solution design for a framed practical problem. As problems can be framed differently, solutions to those problems could also vary. Moreover, to solve a specific problem, a multi-disciplinary knowledge base might be required, which is one of the inherent properties of design science (Simon, 1996). The main difference between this phase and mainstream OSCM research is the 
alternative use of means and ends. In OSCM research domain, means (artifacts-theories) are fixed but ends change, whereas in design science, research means are adaptable or can be invented to serve the purpose(s) of a fixed goal (Cohen, March, \& Olsen, 1972). In the solution refinement phase, several iterations of the proposed solution are tested in a trial and error manner. This eventually results into a cycle of solution design improvement, implementation, and evaluation to obtain the best achievable solution design. Reporting on and recording the components of the solution design that culminated in unintended and erroneous consequences is also crucial (Dennett, 2004). The main takeaway of this phase from the perspective of meansend analysis is that the properties of the artifacts to be designed could change as the research proceeds with no ex-ante predictions. This necessarily does not comply with the straightforward and predefined theoretical consistency, which is common in today's OM research (Holmström et al., 2009). In other words, the design solution and its refinement are exploratory and abductive in nature, whereas the theory-driven approach in OSCM mainstream research is explanatory and inductive. Another critical issue to consider at this stage is to include all the stakeholders (people/organization) and their interests while designing the solution.

Aligned with the design and rigor cycles, the artifact is tested and refined multiple times and the best design solution is chosen. Upon achieving valid results that could be predictive of patterns in subsequent sets of empirical data, a new theory or a variation of the existing theory is proposed and tested in the design cycle. The validation process could be undertaken by several consecutive studies in any given amount of time. This is referred to as the third phase of the design science research methodology by Holmström et al. (2009) as substantive theory only applicable to a narrow context in practice through which the theory has been extracted (Glaser \& Strauss, 1967). Currently, one of the main problems in OSCM research is that many models and theories are substantial; meaning they are suggested but never tested sufficiently 
to yield a validated theory that is generalizable across different contexts of application (Schroeder, 2008). Therefore, the next phase in relevance and design cycles pertains to developing a formal theory by the design scientist. OSCM research has been hitherto mostly concerned with theory testing rather than theory building. First and foremost, the originality and utility of the proposed theory (Lewis \& Grimes, 1999) should be considered by OSCM researchers. The originality of the theory refers to the extent of the theory advancing the knowledge base, which could be, in turn, incremental or revelatory. The utility feature of a formal theory ascertains that the theory is applicable both scientifically and practically (Lewis \& Grimes, 1999). These considerations guide the design scientists to borrow from the knowledge base and use the rigor cycle along with relevance and design cycles to establish the originality and utility of the theory.

\section{Rigor cycle and multi-methods}

Originally, three main research methodologies are used by OSCM researchers. These methodologies include analytical modeling (i.e., application of mathematical, operations research, and simulation models), quantitative empirical research (surveys using questionnaires or interviews), and case study research. Among the prestigious OSCM journals, each of these research methodologies are favored differently. For instance, while Management Science, Production and Operations Management, and Manufacturing \& Service Operations Management outlets prefer studies with an analytical modeling base for the choice of the methodology, Academy of Management Journal and Journal of Operations Management favor empirical research and quantitative form of analysis, including hypothesis development and testing as the primary methodology of choice for the OSCM researchers.

Despite having its merits, focusing on only one method of research tends to confine both the researcher and the scope and potential findings of OSCM research to only a proportion of the actual contributions that a specific research could offer (Singhal \& Singhal, 2012b). On the 
contrary, combining different research methodologies could both maintain research integrity and its links with the practical side of the research (Simchi-Levi, 2014). In some cases, such as in the emerging field of behavioral operations and supply chain management, the use of multimethods is inevitable since usually the developed analytical model of human behavior is tested either empirically or using controlled laboratory experiments to test the validity and applicability of the analytical model (Bendoly, Croson, Goncalves, \& Schultz, 2010; Bendoly, Donohue, \& Schultz, 2006).

In addition to mainstream research methods and their combinations as multi-methods, the application of qualitative and exploratory research method alongside other modes of research could also be considered. More specifically, conducting studies with their focus on qualitative case studies can be construed as one of the main contributors to theory building and development (Barratt, Choi, \& Li, 2011). The call for encouraging research based on qualitative case studies is aligned with the increasing need of OSCM research to more rigor and relevance in empirical research (Boyer, Swink, \& Rosenzweig, 2005; Fisher, 2007). In summary and from the means-end perspective, using multi-methods could facilitate and expedite reaching the optimal design solution that could best serve the purposes of rigor cycle specifically and theory-building in general. In the following sections of this paper, we include some examples from various fields of OSCM research with the aim of improving the quality of research with regards to theory building and practical application using the three cycles of design science.

\section{The application of design science in OSCM research}

In this section, we investigate the applicability of design science methodology in addressing the underlying behavioral causes of the bullwhip effect. The bullwhip effect is the order oscillation and amplification and therefore inventory swings from downstream to upstream supply chain tiers (Lee, 1997). The bullwhip effect could result in many undesirable 
consequences, such as excessive inventory, transportation disruption, lost revenues and stockouts, among others, causing excessive costs in global supply chains (Sheffi, 2015).

The bullwhip effect is the result of both operational and behavioral causes. Some operational sources that cause the bullwhip effect are price variation, order batching, demand signal processing and rationing game caused by shortages (Lee, 1997). In addition to these operational causes, some solutions - such as everyday low pricing, using third party logistics, vendor managed inventory and information sharing - can also mitigate the bullwhip effect (Lee, 1997; Lee, Padmanabhan, \& Whang, 2004). These operational solutions, along with training, are shown to be insufficient to completely eliminate the bullwhip effect (e.g., Tokar, Aloysius, \& Waller, 2012; Wu \& Katok, 2006), and therefore the behavioral causes of the bullwhip effect should be taken into account (Croson, Donohue, Katok, \& Sterman, 2014).

Scholars have attempted to link the bullwhip effect to a variety of behavioral anomalies (e.g., Croson \& Donohue, 2006; Croson et al., 2014; Narayanan \& Moritz, 2015; Sterman, 1989), aiming to address human cognitive limitations and their effect on inventory decisions, and hence the bullwhip effect. Croson et al. (2014) examined the role of coordination risk as a possible behavioral cause of the bullwhip effect. Using a set of laboratory experiments, they found that, even when all the environmental and operational causes of the bullwhip effect are absent, coordination risk could be construed as a trigger of order oscillation and amplification in supply chains. Croson et al. (2014) suggest the addition of coordination stock, establishment of trust between supply chain decision makers and creation of common knowledge to mitigate the bullwhip effect. Narayanan and Moritz (2015) investigated how the cognitive profile of decision makers could be an underlying cause for the bullwhip effect. They find that, even when operational causes of the bullwhip effect are mitigated, participants with tendency to underweight the supply line showed lower levels of cognitive ability. Tokar, Aloysius, Waller, and Hawkins (2015) conducted two studies to evaluate the 
role of framing effect in ordering decisions. The first study contained experiments that examined all forms of framing effect by Levin, Schneider, and Gaeth (1998) in a basic inventory context. Their findings of the first study, which showed the presence of the framing effect in all the single-shot experiments, motivated their second study that investigated the same effect in multi-echelon supply chains. Similar to their first study, the presence of framing effect in multi-echelon supply chains was confirmed in the second study. Subsequently, Tokar et al. (2015) found that the subjects of their experiments were inclined to hold less than optimum inventory, which they showed could be eliminated by framing the same ordering decision in terms of losses. This implied to the role of loss aversion in tuning the ordering quantity. They also predict that if all the prospects are losses, a "superior decision making" should be observed than when prospects introduce gains to supply chains.

Despite the abundance of the aforementioned studies that strive to identify behavioral anomalies affecting bullwhip effect in supply chains, it is clear that the various results obtained from different experiments make it difficult to converge to the main behavioral causes of the bullwhip effect. It is proposed that the application of the proposed design science methodology to this context could direct these scattered attempts into a coherent whole and toward the ultimate behavioral causes of the bullwhip effect:

Relevance and design cycles (solution incubation): So far the framing of the behavioral causes of bullwhip effect in the literature has been merely confined to merely the problem definition and not problem solving. Studies originally assume a subjective set of behavioral causes of these phenomena and conduct behavioral experiments to test their hypothesized results. However, another way of looking at this problem could be to consider de-biasing solutions by giving participants hints to delineate the possible mistakes they might be making due to a particular behavioral anomaly, and how to avoid them (Kaufmann, Michel, \& Carter, 2009; Tokar et al., 2012). Thus, the changes in the performance of participants could then be 
recorded and compared to groups of participants that were unaware of their behavioral regularities. To choose the best sets of de-biasing strategies for the possible behavioral causes of the bullwhip effect, researchers should be familiar with the bodies of knowledge in OSCM and psychology. Moreover, researchers could employ an abductive manner of reasoning (Peirce, 1878; Poole, 1989) and try to understand the possible mix of the behavioral causes of bullwhip effect and the most effective solutions for them, considering their observations from the behavioral experiments.

Relevance and design cycles (solution refinement): The proposed set of experimental frames and the de-biasing strategies developed in the solution incubation phase should first be tested empirically. Next, all the significant and insignificant results gathered from the experiments should be reported as to (1) the behavioral anomalies that were most compliant with the predictions of the analytical models, and (2) the de-biasing strategies that were most effective in regulating the performance of the participants after they have been educated about that particular behavioral anomaly, and how to avoid it. Here, the rigor cycle comes to play as it helps to consolidate the mathematical modeling and empirical testing of the model. Wellinformed conclusions may then be drawn by means of abductive reasoning and making a link between data and the model.

Behavioral operations are, by nature, multi-method research efforts and usually require mathematical models and empirical testing at the same time to prove or disprove assumptions. In this phase, and as the empirical testing of the analytical models proceed, the researchers may come to the understanding that further adjustments to their experiments or their hypotheses are required for achieving a more applicable result to general cases. For instance, the bullwhip effect is usually tested using the traditional beer game as a well-known multi-echelon simulation that replicates real-world supply chains, while being useful for testing behavioral factors of decision making in supply chains (Croson et al., 2014). 
However, the traditional beer game only represents the loss factors (i.e., inventory holding and backorder costs) in supply chain inventory and does not include gains from selling commodity. The lack of the revenue parameter in the game could be a source of concern as the predictions of the analytical models and subsequently the participants' ordering behavior might change as a result of introducing this new parameter.

Design and rigor cycles (substantive and formal theory): As soon as the required solution refinements are carried out and unintended consequences are eliminated, the solution design is complete (Holmström et al., 2009). Next, an OSCM design scientist should cover the theory building phases, given the design solution(s) achieved from the previous phases. The OSCM design scientist will take the suggested theory beyond the pragmatic applications of the theory to a specific context. More specifically, which theoretical discourses could benefit from the substantive theory? This entails systematically introducing the theory in several other contexts and evaluating its credibility (Dubois \& Gadde, 2002). Thus, the proposed theory on the behavioral cause(s) of the bullwhip effect and their debasing solutions should be tested in various relevant experiments with a variety of participants to confirm the assumptions of the substantive theory.

To make the proposed substantive theory a formal theory, it is necessary to achieve generalizability and be applicable to various contexts. One way of doing this is to test the proposed behavioral causes of the bullwhip effect in a broader context of ordering misbehaviors and inventory management in companies, which encompasses the realm of Operations Management. If the theory could be applied to these contexts and explain the behavioral regularities of ordering and their solutions using the substantive theory, then it can be claimed that a formal theory is developed. 


\section{CONCLUSION}

Theory building and making a link between academia and practice is one of the most crucial goals of the OSCM discipline to be achieved. In this conceptual paper, we aimed to examine this issue by emphasizing the applications of the design science research methodology to OSCM research for a systematic way of building formal and practical theories.

Our framework consisted of three main interconnected cycles of relevance, design, and rigor, and entailed testing a design solution to OSCM research problems by using a multimethod approach and drawing conclusions and theories from it. More specifically, we applied the proposed framework to the field of behavioral OSCM and the behavioral causes of the bullwhip effect and showed how the dispersed but growing research efforts in this area could be aligned into drawing a behavioral theory of the ordering preferences of decision makers in supply chains and in organizations. We have also emphasized the applications of multimethods and a multi-disciplinary view of the OSCM problems that could generate more generalizable and effective solutions.

We believe our proposed framework could be starting point into making theory-building practices in OSCM more organized. We would also like to encourage the researchers to follow the proposed procedure recommended in this paper in developing and conducting more holistic research with findings that could be applied in various contexts. Moreover, we would propose that OSCM researchers embrace abductive methods of research rather than only the more common inductive or deductive research, as abductive reasoning could substantially assist OSCM research in the future to make a more robust link between theory and practice. 


\section{References}

Barratt, M., Choi, T. Y., \& Li, M. 2011. Qualitative case studies in operations management: Trends, research outcomes, and future research implications. Journal of Operations Management, 29(4): 329-342.

Bendoly, E., Donohue, K., \& Schultz, K. L. 2006. Behavior in operations management: Assessing recent findings and revisiting old assumptions. Journal of Operations Management, 24(6): 737-752.

Bendoly, E., Croson, R., Goncalves, P., \& Schultz, K. 2010. Bodies of Knowledge for Research in Behavioral Operations. Production and Operations Management, 19(4): 434-452.

Boyer, K. K., Swink, M., \& Rosenzweig, E. D. 2005. Operations Strategy Research in the POMS Journal. Production and Operations Management, 14(4): 442-449.

Boyer, K. K. \& Swink, M. L. 2008. Empirical Elephants - Why multiple methods are essential to quality research in operations and supply chain management. Journal of Operations Management, 26: 337 348.

Choi, T.-M., Cheng, T. C. E., \& Zhao, X. 2016. Multi-Methodological Research in Operations Management. Production and Operations Management, 25(3): 379-389.

Cohen, M. D., March, J. G., \& Olsen, J. P. 1972. A garbage can model of organizational choice. Administrative Science Quarterly, 17(1): 1-25.

Croson, R. \& Donohue, K. 2006. Behavioral Causes of the Bullwhip Effect and the Observed Value of Inventory Information. Management Science, 52(3): 323-336.

Croson, R., Donohue, K., Katok, E., \& Sterman, J. D. 2014. Order Stability in Supply Chains: Coordination Risk and the Role of Coordination Stock. Production and Operations Management, 23(2): 176-196.

Dennett, D. C. 2004. Freedom evolves: Penguin UK.

Dubois, A. \& Gadde, L.-E. 2002. Systematic combining: an abductive approach to case research. Journal of Business Research, 55(7): 553-560.

Finne, M. \& Holmström, J. 2013. A manufacturer moving upstream: triadic collaboration for service delivery. Supply Chain Management: An International Journal, 18(1): 21-33.

Fisher, M. 2007. Strengthening the Empirical Base of Operations Management. Manufacturing $\boldsymbol{E}$ Service Operations Management, 9(4): 368-382.

Glaser, B. G. \& Strauss, A. L. 1967. The Discovery of Grounded Theory: Strategies for Qualitative Research. New York, NY.: Aldine De Gruyter.

Gregor, S. 2006. The nature of theory in information systems. MIS Quarterly, 30(3): 611-642.

Gulati, R. 2007. Tent Poles, Tribalism, and Boundary Spanning: The Rigor-Relevance Debate in Management Research. Academy of Management Journal, 50(4): 775-782.

Hevner, A. R., March, S. T., Park, J., \& Ram, S. 2004. Design science in information systems research. MIS Quarterly, 28(1): 75-105.

Hevner, A. R. 2007. A three cycle view of design science research. Scandinavian Journal of Information Systems, 19(2): 87-92.

Holmström, J., Ketokivi, M., \& Hameri, A.-P. 2009. Bridging Practice and Theory: A Design Science Approach. Decision Sciences, 40(1): 65-87.

Holmström, J. \& Romme, G. 2012. Exploring the future of operations management: Toward an innovation mindset among practitioners and researchers. Journal of Operations Management: Operations \& Supply Management Forum, Essay 15.

Kaufmann, L., Michel, A., \& Carter, C. R. 2009. Debiasing Strategies in Supply Management DecisionMaking. Journal of Business Logistics, 30(1): 85-106.

Lee, H. L. 1997. The bullwhip effect in supply hains. MIT Sloan Management Review, 38(3): 93-102.

Lee, H. L., Padmanabhan, V., \& Whang, S. 2004. Information Distortion in a Supply Chain: The Bullwhip Effect. Management Science, 50(12_supplement): 1875-1886.

Levin, I. P., Schneider, S. L., \& Gaeth, G. J. 1998. All Frames Are Not Created Equal: A Typology and Critical Analysis of Framing Effects. Organizational Behavior and Human Decision Processes, 76(2): 149-188.

Lewis, M. W. \& Grimes, A. I. 1999. Metatriangulation: Building Theory from Multiple Paradigms. Academy of Management Review, 24(4): 672-690. 
March, S. T. \& Smith, G. F. 1995. Design and natural science research on information technology. Decision Support Systems, 15(4): 251-266.

Narayanan, A. \& Moritz, B. B. 2015. Decision Making and Cognition in Multi-Echelon Supply Chains: An Experimental Study. Production and Operations Management. n/a-n/a.

Peirce, C. S. 1878. Deduction, induction, and hypothesis. Popular Science Monthly, 13: 470-482.

Poole, D. 1989. Explanation and prediction: an architecture for default and abductive reasoning. Computational Intelligence, 5(2): 97-110.

Sanders, N. R. \& Wagner, S. M. 2011. Multidisciplinary and Multimethod Research for Addressing Contemporary Supply Chain Challenges. Journal of Business Logistics, 32(4): 317-323.

Schroeder, R. G. 2008. Introduction to the Special Issue on Theory Development in Operations Management. Production and Operations Management, 17(3): 354-356.

Sheffi, Y. 2015. China's Slowdown: The First Stage of the Bullwhip Effect, Harvard Business Review. https://hbr.org/2015/09/chinas-slowdown-the-first-stage-of-the-bullwhip-effect.

Simchi-Levi, D. 2014. OM Forum-OM Research: From Problem-Driven to Data-Driven Research. Manufacturing \& Service Operations Management, 16(1): 2-10.

Simon, H. A. 1996. The sciences of the artificial. Cambridge, MA: MIT Press.

Singhal, K. \& Singhal, J. 2012a. Imperatives of the science of operations and supply-chain management. Journal of Operations Management, 30(3): 237-244.

Singhal, K. \& Singhal, J. 2012b. Opportunities for developing the science of operations and supply-chain management. Journal of Operations Management, 30(3): 245-252.

Singhal, K., Sodhi, M. S., \& Tang, C. S. 2014. POMS Initiatives for Promoting Practice-Driven Research and Research-Influenced Practice. Production and Operations Management, 23(5): 725-727.

Sodhi, M. S. \& Tang, C. S. 2014. Guiding the next generation of doctoral students in operations management. International Journal of Production Economics, 150: 28-36.

Sterman, J. D. 1989. Modeling Managerial Behavior: Misperceptions of Feedback in a Dynamic Decision Making Experiment. Management Science, 35(3): 321-339.

Tokar, T., Aloysius, J. A., \& Waller, M. A. 2012. Supply Chain Inventory Replenishment: The Debiasing Effect of Declarative Knowledge. Decision Sciences, 43(3): 525-546.

Tokar, T., Aloysius, J., Waller, M., \& Hawkins, D. L. 2015. Exploring Framing Effects in Inventory Control Decisions: Violations of Procedure Invariance. Production and Operations Management. $\mathrm{n} / \mathrm{a}-\mathrm{n} / \mathrm{a}$.

van Aken, J. E. 2004. Management Research Based on the Paradigm of the Design Sciences: The Quest for Field-Tested and Grounded Technological Rules. Journal of Management Studies, 41(2): 219246.

van Aken, J. E. 2005. Management Research as a Design Science: Articulating the Research Products of Mode 2 Knowledge Production in Management. British Journal of Management, 16(1): 19-36.

Walker, H., Chicksand, D., Radnor, Z., \& Watson, G. 2015. Theoretical perspectives in operations management: an analysis of the literature. International Journal of Operations $\boldsymbol{\&}$ Production Management, 35(8): 1182-1206.

Wu, D. Y. \& Katok, E. 2006. Learning, communication, and the bullwhip effect. Journal of Operations Management, 24(6): 839-850. 